\title{
1 Supplemental method
}

2

\section{Cloning of wild-type and 13del mutant Col10a1 gene into pcDNA4/TO}

The wild-type CollOal coding sequence (cds) plus 149 bp 3' untranslated region (UTR) was previously cloned into the vector pCEP4-BM40-mycEK (1). The BM40 signal peptide, $c$-myc tag, enterokinase cutting site and the wild type Colloal gene was amplified from the Colloal-pCEP4-BM40-mycEK plasmid using primers (5'GCTTGATATCGTCGACGCCTGCCGCCTGCCGGC-3' ${ }^{\prime}$ and AgGCCGCGGTGATCAGCGGCCGCGGAAACTTGGTCCCAGCGTGC-3'). The PCR product was cloned into EcoRV and NotI site of pcDNA4/TO (Invitrogen). To generate the 13del-CollOal-pcDNA4/TO construct, the 13del Colloal was amplified from 13delpBluescript II SK(-) using the abovementioned primers. The XhoI and NotI (2) fragment containing the mutated region was then cloned into the $w t$-CollOal-pcDNA4/TO plasmid to produce the 13del-CollOal-pcDNA4/TO plasmid.

\section{Cell line maintenance and induction of differentiation}

The ATDC5 cells were maintained as described by Shukunami C et al (3). The parental ATDC5 cells were grown in the basal medium which is DMEM/F12 (Cat. \#12400-024, Invitrogen-Gibco) containing $10 \mu \mathrm{g} / \mathrm{ml}$ human transferrin (Cat. \# T8158, Sigma) and $5 \mathrm{ng} / \mathrm{ml}$ sodium selenite (Cat. \# S5261, Sigma) supplemented with 5\% fetal bovine serum (Biosera), penicillin/streptomycin (Invitrogen-Gibco) and fungizone (Invitrogen-Gibco) at $37^{\circ} \mathrm{C}$ under $5 \% \mathrm{CO} 2$. To induce differentiation, cells were firstly plated at $70 \%$ confluence and $10 \mu \mathrm{g} / \mathrm{ml}$ bovine insulin (Cat. \# I6634, Sigma) was added when the cells were confluent. In the whole course of culture, medium was replenished every two to three days. 
ATDC5 cells were plated in $35 \mathrm{~mm}$ dishes and were induced to differentiate. At each time point, cells were washed with PBS and fixed with absolute methanol at $-20 \mathrm{oC}$ for 2 min. Cells were then stained with $0.1 \%$ (w/v) Alcian blue (Cat. \# A5268, Sigma) solution (in 0.1N $\mathrm{HCl})$ at $4^{\circ} \mathrm{C}$ overnight. Stained cultures were washed with MilliQ water thoroughly to remove excessive stain.

\section{Western blot analysis}

To prepare whole cell lysate, cultured cells were washed with PBS and lysed in $130 \mu$ lysis buffer [50mM Tris- $\mathrm{HCl}(\mathrm{pH} 8.0), 150 \mathrm{mM} \mathrm{NaCl}, 1 \%(\mathrm{v} / \mathrm{v}) \mathrm{NP}-40,0.5 \%$ (w/v) sodium deoxycholate, $0.1 \%(\mathrm{w} / \mathrm{v}) \mathrm{SDS}$ ] for each well. The whole cell lysates were collected and stored at $-20^{\circ} \mathrm{C}$. For the analysis of collagen $\mathrm{X}$ protein from culture medium, medium was replenished when the cells were at $70 \%$ confluence. After further culture for two days, medium was harvested and an equal volume of 50\% ammonium sulfate was added. Proteins were precipitated at $4^{\circ} \mathrm{C}$ overnight with mixing. Precipitated proteins were recovered by centrifugation at 13,400 rpm for $30 \mathrm{~min}$. The protein pellet was dissolved in the SDS-PAGE loading buffer. $20 \mu \mathrm{g}$ of whole cell lysate or precipitated protein obtained from $300 \mu$ l culture medium was loaded into an $8 \%$ polyacrylamide gel. Exogenous collagen $\mathrm{X}$ proteins were detected by anti-c-myc antibodies (Cat. \# sc-798, Santa Cruz Biotechnology). $10 \mu \mathrm{g}$ of proteins extracted and quantified from wt and 13del HCs were loaded into an $8 \%$ polyacrylamide gel.

\section{Immunostaining}

ATDC5 cells were plated on cover slips and cells were allowed to attach overnight. Cultured 
51 CollOal gene. Cells were fixed with absolute methanol $24 \mathrm{~h}$ post-transfection. Over-

52 expressed collagen X proteins were detected by anti-c-myc antibodies (Cat. \# sc-798, Santa Cruz Biotechnology). Nuclei were detected with DAPI staining of DNA. The fluorescent microscopic images of wt and 13del samples were captured with Olympus BX51 using exactly the same parameters (green channel: 488nm, exposure $800 \mathrm{~ms}$, red channel: $555 \mathrm{~nm}$, exposure 50ms; blue channel: 405nm, exposure 50ms).

\section{Isolation and maintenance of stable cell lines}

ATDC5 cells in undifferentiated culturing condition were plated in a 6-well plate with a cell density of $5 \times 10^{4}$ cells/ well. $1 \mu \mathrm{g}$ of $w t$-CollOal-pcDNA4/TO or 13del-CollOalpcDNA4/TO plasmid DNA was transfected using GeneJuice® transfection reagent (Novagen) the day after plating. Cells in each well were split into a $100 \mathrm{~mm}$ dish after $24 \mathrm{~h}$ and positive transfectants were selected with $150 \mu \mathrm{g} / \mathrm{ml}$ Zeocin (Invitrogen). Culture medium was replenished every two to three days. Colonies were picked after 14 days of selection. Clones were expanded, maintained and differentiated in medium containing $150 \mu \mathrm{g} / \mathrm{ml}$ Zeocin hereafter. For mass spectrometry analysis proteins were isolated, reduced, trypsin digested from wt and 13del ATDC5 cells.

\section{In vitro proteomic analysis}

Protein sample preparation and iTRAQ labeling - The wt and 13del stable cell lines were plated in $100 \mathrm{~mm}$ dishes and induced to differentiate for 15 days as described above. The cultured cells were harvested and cells were resuspended in $300 \mu 1$ dissolution buffer from the iTRAQ Reagent 8-plex Kit (Applied Biosystems) with 0.1\% SDS. Cells were lysed by sonication followed by centrifugation at $13,400 \mathrm{rpm}$ at $4^{\circ} \mathrm{C}$ for $1 \mathrm{~h}$. The soluble fraction was kept and protein concentration was determined by bicinchoninic acid (BCA) assay. $100 \mu \mathrm{g}$ 
protein from each condition was reduced and alkylated followed by overnight trypsin digestion before iTRAQ labeling (isobaric tags 119,116 and 116,113 labeling 13del and wt respectively, were used in two experiments).

LC-MS/MS analysis - The iTRAQ labeled peptides from the two samples were pooled together and separated by the PolySULFOETHYL $\mathrm{A}^{\mathrm{TM}}$ column $(200 \mathrm{~mm} \times 2.1 \mathrm{~mm}$ i.d., 5 $\mu \mathrm{m}, 300 \AA$, PolyLC) for the first dimension strong cation exchange chromatography. Peptides were eluted in a linear binary gradient of 150 to $350 \mathrm{mM} \mathrm{KCl}$ with $10 \%$ acetonitrile and $10 \mathrm{mM} \mathrm{KH} 2 \mathrm{PO} 4(\mathrm{pH} \mathrm{3.0)}$ at a flowrate of $200 \mu \mathrm{l} / \mathrm{min}$. Peptides eluted were collected in $200 \mu$ fractions. Some fractions were pooled together according to the elution profile such that different fractions contain comparable amount of peptides. The fractions were freeze dried and then desalted using the Sep-Pak ${ }^{\circledR}$ cartridge. The fractions after desalting were freeze dried and then reconstituted in $20 \mu$ mobile phase A ( $2 \%$ acetonitrile $0.5 \%$ formic acid) for the second dimension reverse phase liquid chromatography. $8 \mu$ l of each fraction was run into an in-house packed C18 (Phenomenex Jupiter) HPLC column $(200 \mathrm{~mm} \times 0.15$ mm i.d., $3 \mu \mathrm{m}, 300 \AA$ ) using the Agilent 1100 capillary LC pump, which was coupled to the QSTAR XL system (Applied Biosystem MDS Sciex). Peptides were eluted into the MS system at a flowrate of $1 \mu \mathrm{l} / \mathrm{min}$ in a linear binary gradient of $5 \%$ to $35 \%$ mobile phase $\mathrm{B}$ ( $98 \%$ acetonitrile, $0.5 \%$ formic acid) for $90 \mathrm{~min}$. Mass spectrometric analysis was performed using the QSTAR XL mass spectrometer which equipped with a electrospray ion source and two quadrupole followed by a reflectron time-of-flight as the mass analyzer. The MS analysis was operated in the information-dependent acquisition (IDA) mode with 1 full scan followed by $5 \mathrm{MS} / \mathrm{MS}$ scans each of $1.5 \mathrm{sec}$ acquisition time (duty cycle of $9 \mathrm{sec}$ ) for the $90 \mathrm{~min}$ RPLC gradient. Full scan mass spectra were acquired from $m / z, 400$ to 1500 . Three to five most abundant ions in this range having an absolute count above 60 and charge states between +2 
and +4 were automatically selected for fragmentation. A dynamic exclusion of $60 \mathrm{sec}$ was applied for ions selected. Rolling collision energy was used and ions $\mathrm{m} / \mathrm{z}$ in the range of 100 to 2000 was screened in MS/MS scans.

Proteomics data analysis and bioinformatics - Spectra obtained from MS/MS were submitted to search against the SwissProt database (release 56.8, Feb 2009 410,518 sequences) using the ProteinPilot 2.0 (Applied Biosystems) using the Paragon algorithm. Proteins were reported with unused protein score above (confidence above 95\%). In experiment I, 18041 unique peptides were matched which contribute to the identification of 589 unambiguous proteins from ProteinPilot program with 95\% confidence. In experiment II, 694 nonredundant proteins were mapped by ProteinPilot from 16661 unique peptides with the same search criteria. Complete list of confident peptides (>95) identified in exp 1 and 2 is shown in Supplementary Table 4C and 4D. In total, 938 unique proteins were identified from the two experiments. Out of 589 and 694 identified proteins from exp I and exp II, 533 and 694 proteins were quantified (Supplementary Table 4A). To obtain a fold change a bias correction was applied by the Protein Pilot to the calculated iTRAQ ratios for each biological replicate (Sup Table 4A). The Error factor represents the variability among the ratios of the peptide assigned to each protein and was calculated by Protein Pilot (Sup Table 4A). An average fold change was calculated and used to select differentially expressed proteins. After selection criteria ( $\log 2 \mathrm{FC} \geq 0.5$ or $\log 2 \mathrm{FC} \leq-0.5$ ) were applied, 77 proteins were identified as upregulated and 118 as downregulated (this include protein identification based on single peptide hits and $\mathrm{P}>0.05)$. Peptide ratios were determined and corrected for any systematic bias. Using the results from the Pro Group ${ }^{\mathrm{TM}}$ Algorithm, an average ratio was calculated for each protein. P-values were computed for each ratio, providing a convenient measure of its statistical significance represented in Supplementary Table 4A. 
127 1. Bengtsson, E.; Aspberg, A.; Heinegard, D.; Sommarin, Y.; Spillmann, D., The amino128 terminal part of PRELP binds to heparin and heparan sulfate. J Biol Chem 2000, 275, (52), 129 40695-702.

$130 \quad 2 . \quad$ Tsang, K. Y.; Chan, D.; Cheslett, D.; Chan, W. C.; So, C. L.; Melhado, I. G.; Chan, T. 131 W.; Kwan, K. M.; Hunziker, E. B.; Yamada, Y.; Bateman, J. F.; Cheung, K. M.; Cheah, K. 132 S., Surviving endoplasmic reticulum stress is coupled to altered chondrocyte differentiation 133 and function. PLOS Biol 2007, 5, (3), e44.

134 3. Shukunami, C.; Shigeno, C.; Atsumi, T.; Ishizeki, K.; Suzuki, F.; Hiraki, Y., 135 Chondrogenic differentiation of clonal mouse embryonic cell line ATDC5 in vitro: 136 differentiation-dependent gene expression of parathyroid hormone (PTH)/PTH-related 137 peptide receptor. J Cell Biol 1996, 133, (2), 457-68. 